\title{
Psychopathology in patients with schizophrenia attending a psychiatry outpatient clinic at a tertiary care hospital
}

\author{
Hiranya Wijesundara, Madhubhashinee Dayabandara, Arjuna Ellepola and Raveen Hanwella
}

\section{Background}

Patients with schizophrenia who are not acutely ill continue to experience different levels of psychopathology, despite a relatively stable lifestyle. Aims

To describe the pattern of symptoms in patients with schizophrenia attending an outpatient clinic using the Brief Psychiatric Rating Scale Expanded version (BPRS-E).

\section{Methods}

Consecutive patients in an outpatient clinic, with a diagnosis of schizophrenia were assessed. Analysis was based on the four-factor cluster of symptoms: thought disturbance, animation, mood disturbance and apathy identified using the BPRS-E.

Results

Sample size was 178 (males $54.5 \%$, mean age = 39.29 years, mean illness duration $=13.29$ years, mean total BPRS score $=32.11$ ). Apathy had the highest mean score among the symptom clusters
(6.54, SD 3.56), while blunted affect (1.87, SD 1.23) and emotional withdrawal (1.85, SD 1.26) had highest individual item mean scores. Patients on clozapine had significantly more symptoms of thought disturbance and animation compared to others, $(P<0.05)$. There was no significant difference in symptoms between patients on typical and atypical antipsychotics. Those on higher doses of clozapine $(>400 \mathrm{mg}$ ) had significantly higher scores in thought disturbance and apathy. Higher scores on total BPRS, negative symptoms and thought disturbance were associated with lower levels of functioning.

\section{Conclusions}

Patients with schizophrenia on treatment continue to have symptoms. Negative symptoms are the most predominant. Patients on clozapine have a higher level of psychopathology. The degree of negative symptoms and thought disturbance had a significant association with the level of functioning.

SL J Psychiatry 2011; 2 (1):23-27

\section{Introduction}

Schizophrenia is a psychiatric illness with varied psychopathology and several dimensions of symptoms. In addition to positive and negative symptom dimensions which have been described for decades, affective, cognitive and aggressive symptom dimensions are now recognised $(1,2)$. Positive symptoms such as delusions and hallucinations are easily identified since they are seen in most acutely ill patients and lead to disturbances in behaviour. These symptoms usually respond to antipsychotic medication. In contrast, negative symptoms such as avolition and blunted affect, which have a chronic course and respond poorly to medication, have a significant impact on the social and occupational functioning and the overall outcome of the patient. Therefore detection and treatment of negative symptoms is now recognised as an important aspect in the management of schizophrenia. Combinations of pharmacological and psychosocial interventions such as rehabilitation are effective in the treatment of these symptoms $(1,3)$. In recent years, research has focused on discovering medication to treat cognitive deterioration in schizophrenia as there is evidence that this has a significant impact on the outcome of the illness. Therefore the assessment of different symptom dimensions of patients with schizophrenia is relevant in planning their long term management.

For the objective assessment of psychopathology in research as well as in clinical practice, various tools such as the Brief Psychiatric Rating Scale (BPRS), the
Positive and Negative Symptom Scale (PANSS), the Scale for Assessment of Negative Symptoms (SANS) and the Scale for Assessment of Positive Symptoms (SAPS) are used. The BPRS, one of the three main symptom rating scales used in schizophrenia, was developed in 1962 (4). It was initially a 16/18-item scale which was rated using both observations of the patient and the patient's self-reports. Though BPRS is not specific to schizophrenia, it assesses a range of positive, negative, affective and behavioural symptoms. Recently it has been expanded to 24 items (BPRS-E), making it more comprehensive in the assessment of negative and affective symptoms (5). The BPRS also has excellent inter-rater reliability and high sensitivity to change and has been extensively used in many diagnostic groups and for a wide range of purposes (5).

Patients with chronic schizophrenia attending follow up clinics are mostly in remission but some patients continue to have varying degrees of psychopathology, despite having a relatively stable lifestyle. The type of antipsychotic used, dose, compliance and illness related factors all influence the level of symptoms in these patients. Two internation studies on the outcome of schizophrenia have found that patients have a better outcome in developing countries compared to developed countries $(6,7)$. As there is little data available on the outcome of patients with schizophrenia in Sri Lanka we studied the psychopathology of patients with schizophrenia attending a follow up clinic. 


\begin{tabular}{|lrc|}
\hline \multicolumn{3}{|l|}{ Table 1- Demographic and illness characteristics } \\
\hline Employment & $\begin{array}{l}\text { Frequency } \\
(\mathrm{n}=178)\end{array}$ & Percentage \\
\hline Age distribution & 2 & 1.1 \\
12-19 years & 100 & 56.2 \\
20-40 years & 67 & 37.6 \\
$41-60$ years & 9 & 5.1 \\
$>60$ years & & \\
\hline Illness duration & 35 & 19.7 \\
$<5$ years & 87 & 48.9 \\
$5-15$ years & 56 & 31.5 \\
$>15$ & & \\
\hline Duration of current & & \\
treatment & 118 & 66.3 \\
$0-5$ & 47 & 26.3 \\
$5-15$ & 13 & 7.3 \\
$>15$ &
\end{tabular}

\section{Methods}

Sample included one hundred and seventy eight consecutive patients with schizophrenia, attending follow up clinics over a period of one month. All patients with schizophrenia attending the clinic during this period were recruited except those with learning disability and those who refused consent to participate in the study.

Demographic and illness data was collected using an interviewer administered questionnaire completed by the researchers. Demographic data collected included clinic number, age, gender, data regarding the illness and antipsychotic medication (name, dose and duration).

The severity of illness was assessed using the BPRS-E, a 24 -item scale with a scoring range from 1 (not present) to 7 (extremely severe). The possible total score ranges from 24 to 168 . The original 18 items are somatic concern, anxiety, depression, guilt, hostility, grandiosity, suspiciousness, hallucinations, unusual thought content, disorientation, conceptual disorganization, blunted affect, emotional withdrawal, motor retardation, tension, uncooperativeness, excitement, mannerisms and posturing. The six new items in the expanded version (self neglect, suicidality, bizarre behavior, distractibility, motor hyperactivity, elated mood) have improved its ability to assess negative and mood symptoms further $(8,9)$. The researchers were trained in the use of the BPRS-E.

The level of functioning was assessed using the

\begin{tabular}{|lcc|}
\hline \multicolumn{3}{|l|}{ Table 2- Level of psychopathology measured by BPRS-E } \\
\hline BPRS-E Scores & $\begin{array}{c}\text { Frequency } \\
(n=178)\end{array}$ & Percentage \\
\hline 24 & 42 & 23.6 \\
$25-36$ & 95 & 53.4 \\
$37-48$ & 28 & 15.7 \\
$49-60$ & 8 & 4.5 \\
$61-72$ & 4 & 2.2 \\
$73-84$ & 1 & 0.6 \\
$>84$ & 0 & 0 \\
\hline
\end{tabular}

\begin{tabular}{|lll|}
\hline \multicolumn{3}{|l|}{ Table 3- Mean scores of symptom clusters } \\
\hline Symptom cluster & Mean score & SD \\
Thought disturbance & 5.57 & 2.44 \\
Animation & 4.54 & 1.29 \\
Mood disturbance & 5.61 & 2.69 \\
Apathy & 6.54 & 3.56 \\
\hline
\end{tabular}

Activities of Daily Living Scale (ADLS) (10). Both basic activities of daily living (ADL) which include bathing, continence, dressing, feeding, toileting and transferring and instrumental ADL which consisted of using the telephone, travelling, shopping, preparing meals, house work, managing medication and managing finances were assessed. Patients' level of functioning was scored on a scale of 0-3 (does not carry out, dependent, need assistance, independent). The possible range of scores was 0-18 for basic ADL and 0-21 for instrumental ADL. Statistical analysis of data was carried out using SPSS- version 14.0. Independent sample $t$ test was used to compare means between two groups.

\section{Results}

\section{Demographic and illness characteristics}

There were 97 males (54.5\%) and 81 (45.5\%) females in the sample. The demographic and illness characteristics are given in table 1 . The mean age of the sample was 39.3 years $(\mathrm{SD}=11.25)$. A majority $(56.2 \%)$ of the patients were aged between 20-40 years. The mean duration of illness was 13.3 years $(\mathrm{SD}=8.8)$. Thirty five patients $(19.7 \%)$ had a duration of illness of less than 5 years. Nearly half of the sample (48.9\%) had the illness for 5-15 years. Nearly a third of the patients had the illness for over 15 years.

In this sample, 75 patients $(42.13 \%)$ were treated with clozapine, $60(33.71 \%)$ with either risperidone or olanzapine, while $43(24.16 \%)$ were treated with typical antipsychotics. The mean duration of current antipsychotic treatment was 5.4 years. Mean clozapine dose was $387.3 \mathrm{mg}$ (dose range $150-800 \mathrm{mg}$ ).

\section{Level of psychopathology}

Level of psychopathology as assessed by the BPRS-E is given in table 2. In the sample, the total BPRS-E scores ranged from 24 to 73 . The possible range was 24 to 168 . The mean was 32.11 ( $S D=9.11)$. Of the patient's in this sample, $23.6 \%$ had a score $\leq 24$ which indicated that they are free from any psychopathology

\begin{tabular}{|c|c|c|c|}
\hline Mean & $\begin{array}{l}\text { Typical } \\
\text { antipsychotics } \\
(\mathrm{n}=43)\end{array}$ & $\begin{array}{l}\text { Atypical } \\
\text { antipsychotics } \\
(\mathrm{n}=60)\end{array}$ & $\begin{array}{l}\text { Significance } \\
P\end{array}$ \\
\hline Total BPRS-E & 29.58 & 30.52 & 0.43 \\
\hline Thought & 5.05 & 5.22 & 0.68 \\
\hline Animation & 4.35 & 4.37 & 0.93 \\
\hline Mood & 5.65 & 5.58 & 0.90 \\
\hline Apathy & 5.84 & 6.40 & 0.34 \\
\hline
\end{tabular}




\begin{tabular}{|c|c|c|c|}
\hline Symptom cluster & $\begin{array}{l}\text { ADSL score } \\
\text { low }\end{array}$ & $\begin{array}{l}\text { ADSL score } \\
\text { medium- } \\
\text { high }\end{array}$ & Significance \\
\hline Total BPRS-E & 38.47 & 29.67 & $p<0.001$ \\
\hline $\begin{array}{l}\text { Thought } \\
\text { disturbance }\end{array}$ & 6.61 & 5.17 & $p<0.001$ \\
\hline Animation & 4.89 & 4.41 & $p=0.02$ \\
\hline Mood disturbance & 5.81 & 5.53 & $p=0.53$ \\
\hline Apathy & 8.40 & 5.83 & $p<0.001$ \\
\hline
\end{tabular}

as measured by the BPRS-E. In $53.37 \%$ of patients, the total BPRS-E scores were within the 25-36 range. When the 24 individual items were analyzed the highest mean scores were for blunted affect $(1.87$; SD $=$ $1.23)$ and emotional withdrawal $(1.85 ; \mathrm{SD}=1.26)$, both of which are negative symptoms. Hallucinations (1.77; $\mathrm{SD}=1.53)$, somatic concerns $(1.69 ; \mathrm{SD}=1.22)$ and suspiciousness $(1.61 ; \mathrm{SD}=1.25)$ also had high mean scores.

\section{Mean scores of symptom clusters}

Several factor analysis studies have been done using BPRS to identify symptom clusters in patients with schizophrenia. In a recent study Donell et al. identified four symptom clusters using the BPRS-E (11). These four factors were used for data analysis in this study. The symptom cluster "thought disturbance" included the four items of unusual thoughts, grandiosity, suspiciousness and hallucinations. The symptom cluster "animation" included the items motor hyperactivity, excitement, tension and elated mood. The symptom cluster "mood disturbance" included the items depression, anxiety, guilt and suicidality. The symptom cluster "apathy" included emotional withdrawal, blunted affect, motor retardation and self neglect. Table 3 shows the mean scores of the symptom clusters.

The possible score range for each symptom cluster was from 4 to 28 . The highest mean score was for the apathy cluster $(6.54, \mathrm{SD}=3.56)$ representing negative symptoms followed by mood disturbances and disturbances of thought.

\section{Functional level of patients}

The vast majority of patients $(97.2 \%)$ were independent in all six basic activities of daily living, while $72.5 \%$ were independent in all seven instrumental activities of daily living. The mean of the basic ADLS score was $17.93(\mathrm{SD}=0.42)$ and the mean of the instrumental ADLS score was $19.84(\mathrm{SD}=2.63)$.

Comparison between typical and atypical users There was no statistically significant difference between those treated with atypical antipsychotics (excluding clozapine) and typical antipsychotics for the total mean BPRS-E score or symptom cluster scores (Table 4).

\section{Comparison between clozapine and other antipsychotics}

The mean BPRS-E scores of patients on clozapine were higher (34.83) compared to patients treated with other antipsychotics (30.13) and the difference was statistically significant $(\mathrm{t}=3.18, \mathrm{p}=0.002)$. The mean score in the symptom cluster "thought disturbance" was significantly higher in the clozapine group (6.16) compared with the group on other antipsychotics (5.15) $(\mathrm{t}=2.68, \mathrm{p}=0.008)$. The mean scores in the "animation" symptom cluster was also significantly higher in the clozapine group (4.8) compared to those on other antipsychotics (4.36) $(\mathrm{t}=2.11, \mathrm{p}=0.036)$. The scores for the "mood" and "apathy" clusters were not significantly different between the two groups.

\section{Association between clozapine dose and BPRS-E scores}

Those who were treated with more than $400 \mathrm{mg}$ of clozapine had significantly higher scores of mean BPRS-E $(p=0.003)$ and the means of the symptom clusters of thought disturbance and apathy compared to those on $<400 \mathrm{mg}(\mathrm{p}=0.035$ and $\mathrm{p}=0.20)$.

\section{Correlation with severity of illness}

A study on the correlation of the 18-item BPRS scores with the Clinical Global Impressions Severity Scale (CGI-S) shows that patients with minimum illness on CGI-S scored 31 on the BPRS-E and those with moderate illness on CGI-S scored 41 on the BPRS-E and those with severe illness scored 53 (12).

When the BPRS-E scores in this sample were categorised according to above cut-off values the majority, 148 patients $(83.15 \%)$ were found to score below the cut off score for 'mildly ill' $(<31)$. Of the sample $12.92 \%$ had 'mild illness' (score range 31-41) and $2.81 \%$ had 'moderate illness' (score range 42-52). Only two patients $(1.1 .2 \%)$ had scores of 53 or above $t$ obe categorised as 'severe illness'. This indicates that while patients have residual psychopathology, their illness severity corresponding to CGI-S assessment is low.

\section{Applying remission criteria}

According to the 'Remission in Schizophrenia Working Group' led by Andreason, remission is defined by using an absolute threshold of severity of the diagnostic symptoms of schizophrenia, rather than percentage improvements from a particular baseline. The 18 item BPRS is one of the three validated instruments used to define symptomatic remission in research (13). A score of 3 or less on seven items- (grandiosity, suspiciousness, unusual thought content, hallucinations, conceptual disorganization, mannerisms/ posturing and blunted affect) is required to fulfill the severity criteria for remission. A maintenance period of six months with the above severity of symptoms has been defined as a minimum time threshold.

Applying only the severity criteria (without the time criterion) in this study sample, 149 patients $(83.71 \%)$ were in symptom remission.

\section{Association between BPRS-E scores and Activities of Daily Living}

Table 5 illustrates the mean BPRS-E total and subcale scores according to level of functioning as measured by Activities of Daily Living Scale (ADLS). The ADLS scores if within the lower quartile of total scores, were categorised as low and if within the upper 3 quartiles as 
medium-high. Those with low level of functioning as measured by the ADLS score had significantly higher mean scores on the total BPRS and subscales of thought, animation and apathy subscales. The difference in the mood subscale was not statistically significant.

\section{Discussion}

The results of this study indicate that the majority of patients with schizophrenia have some degree of psychopathology, mostly negative symptoms as represented by the apathy cluster. Mood symptoms were also relatively common, followed by thought disturbances. Patients on typical antipsychotics did not differ significantly in the degree of psychopathology compared to those on atypical antipsychotics. Patients with resistant schizophrenia who were on clozapine had relatively higher levels of psychopathology compared to those on other antipsychotics. The difference was most marked in the symptom clusters representing thought disturbance and behavioural problems. There were significant associations between negative symptoms and thought disturbances and the level of functioning.

The finding that most patients have a significant degree of psychopathology especially negative symptoms despite being on treatment has been reported in other studies (9). A recent study of patients on clozapine in the same setting found that $67.2 \%$ had negative symptoms (10).

An area of interest in recent years has been the concept of remission in schizophrenia. With increasing understanding and expectations related to the long term course of the illness and the evolution of psychosocial and pharmacological therapies, several groups have proposed definitions and criteria for remission in schizophrenia $(13,14,15)$. The BPRS scores have been used in defining symptom criteria in most such instances. In a one year prospective, observational study aimed at standardising remission criteria, researchers found varying remission rates in the range of $70-80 \%$ when different criteria were used. When only a BPRS total score criterion (total score of 36 or less) was applied the remission rate was $78.6 \%$. This declined to $75.0 \%$ when a BPRS psychotic item criterion (a BPRS psychotic item score of 3 or less) was included. As yet, there are no studies which applied the remission criteria developed by the 'Remission in Schizophrenia Working Group'.

Apart from symptoms, aspects that are of importance to patients are the level of functioning and the quality of life. As reported in several studies, psychopathology does affect the level of functioning and quality of life of patients $(16,17,18)$. Impact of affective and negative symptoms on the quality of life has been reported in many studies. Although this study did not measure the quality of life, it found significant associations between level of functioning and symptom clusters of apathy, animation and thought disturbances.

There are several limitations in this study. We have not obtained a random sample of patients for analysis. As such, the sample may not represent the whole population of follow-up patients with schizophrenia attending the clinic. The sample size is not adequate to compare individual antipsychotic medications. As the BPRS does not include the cognitive symptom dimension we have not evaluated this aspect. Our study has not assessed the quality of life, service satisfaction and caregiver burden.

Our results show several significant findings. Since the majority of patients are in remission this indicates that patients attending follow up clinics have good illness control. However this study cannot comment on the overall outcome of patients with schizophrenia which include those who do not attend follow up care. For the minority of patients who have high levels of psychopathology there is a need to consider alternative psychopharmacological treatment and review the effects of such a change. The importance of assessing wider symptom dimensions including negative symptoms at follow up is highlighted in this study. For patients with high levels of negative symptoms, the use of psychosocial rehabilitation interventions in combination with medication should be stressed in the clinical management of patients with schizophrenia to improve their level of functioning.

\section{Conclusions}

Patients with schizophrenia on long term antipsychotic medication attending follow up clinics continue to experience some degree of psychopathology. The negative symptom dimension is more prominent while mood symptoms and thought disturbances are also prevalent. Patients with resistant schizophrenia on clozapine continue to have a higher level of psychopathology than patients on other antipsychotics especially in the symptom clusters of thought disturbance and animation. The degree of negative symptoms and thought disturbance have significant associations with the level of functioning.

\section{Declaration of interest}

None

\section{Hiranya Wijesundara, MBBS,MD(Psych)}

Senior Registrar, University Psychiatry Unit, Colombo, Sri Lanka Madhubhashinee Dayabandara, MBBS,MD(Psych) Lecturer, Department of Psychological Medicine, Faculty of Medicine, University of Colombo, Sri Lanka Arjuna Ellepola, MBBS, MD(Psych) Senior Registrar, University Psychiatry Unit, Colombo, Sri Lanka Raveen Hanwella MBBS,MD(Psych),FRCPsych Senior Lecturer, Department of Psychological Medicine, Faculty of Medicine, University of Colombo, Sri Lanka Corresponding author

Hiranya Wijesundara, University Psychiatry Unit, National Hospital of Sri Lanka, Colombo, Sri Lanka

E mail: hiranya.w@gmail.com 


\section{References}

1. Gelder M, Harrison P, Cowen P. Shorter Oxford textbook of Psychiatry. 5th ed. New York: Oxford University Press, 2006.

2. Stahl SM. Stahl's Essential PsychopharmacologyNeuroscientific Basis and Practical Applications. 3rd ed. Cambridge: Cambridge University Press, 2008.

3. Packer S, Husted J, Cohen S, Tomlinson G. Psychopathology and quality of life in schizophrenia. J Psychiatry Neurosci. 1997; 22(4):231-4.

4. Overall J E \& Gorham D R. The brief psychiatric rating scale. Psychol. Rep. 10:799-812, 1962.

5. Mortimer AM. Symptom rating scales and outcome in schizophrenia. BJPsych. 2007; 191(suppl.50): s7-14.

6. Jablensky A, Sartorius N, Ernberg G, Anker M, Korten A, Cooper JE, Day R, Bertelsen A. Schizophrenia: manifestations, incidence and course in different cultures. A World Health Organization ten-country study. Psychol Med Monogr Suppl 1992; 20:1-97.

7. Leff J, Sartorius N, Jablensky A, Korten A, Ernberg G. The International Pilot Study of Schizophrenia: five-year follow-up findings. Psychol Med 1992; 22(1):131-145.

8. Ventura J, Nuechterlein KH, Subotnik KL, Gutkind D, Gilbert EA. Symptom dimensions in recent-onset schizophrenia and mania: a principal components analysis of the 24-item Brief Psychiatric Rating Scale. Psychiatry Res. 2000 Dec 27; 97(2-3):129-35.

9. Dingemans PM, Linszen DH, Lenior ME, Smeets RM. Component structure of the expanded Brief Psychiatric Rating Scale (BPRS-E). Psychopharmacology (Berl). 1995 Dec; 122(3):263-7.
10. de Silva V, Hanwella R. Efficacy of clozapine in patients with chronic schizophrenia. SL J Psychiatry 2010; 1 (2):47-50.

11. Burlingame GM, Seaman S, Johnson JE et al. Sensitivity to change of the BPRS-E, An item and subscale analysis. Psychological Services. May 2006; Vol 3(2): 77-87.

12. Leucht S, Kane JM, Kissling W et al. Clinical implications of Brief Psychiatric Rating Scale scores. BJPsych 2005:187,366-371.

13. Andreason N, Carpenter WT, Kane JM et al. Remission in Schizophrenia: Proposed criteria and rationale for consensus. AJPsych 2005; 162:441-449.

14. Chung S, Bahk WM, Kwon JS, Kang SH, Joo YH, Ahn JH, Kim CY. Remission Rates and Criteria for Clinical Studies on Schizophrenia. Korean J Psychopharmacol. 2007 Jul; 18(4):231-239.

15. Curtis CE, Calkins ME, Grove WM, Feil KJ, Iacono WG: Saccadic disinhibition in patients with acute and remitted schizophrenia and their first-degree biological relatives. AJPsych 2001; 158:100-106.

16. Becker T, Knapp M, Knudsen HC. et al. The EPSILON Study of schizophrenia in five European countries: design and methodology for standardizing outcome measures and comparing patterns of care and service costs. BJPsych 1999: 175: 514-52.

17. Zahid MA, Ohaeri JU, Elshazly AS, Basiouny MA, Hamoda HM, Varghese R. Correlates of quality of life in an Arab schizophrenia sample. Soc Psychiatry Psychiatr Epidemiol. 2010 Sep; 45(9):875-87.

18. Huppert JD, Weiss KA, Lim R, Pratt S, Smith TE. Quality of life in schizophrenia: contributions of anxiety and depression. Schizophr Res. 2001 Sep 1; 51(23):171-80. 